\title{
Case Report: A rare case of prosthetic valve infective endocarditis caused by Aerococcus urinae [version 1; peer
}

\section{review: 2 approved with reservations]}

\section{Muhammad Adeel (D1), Saman Tariq2, Hisham Akthar (iD)2, Ahmed Zaghloul3, Corina Iorgoveanu (D)3, Carina Dehner(iD)}

${ }^{1}$ Yale New Haven Health, Bridgeport Hospital, Bridgeport, CT, USA

${ }^{2}$ Galway University Hospital, Galway, Ireland

${ }^{3}$ University of Connecticut Health Center, Farmington, CT, USA

${ }^{4}$ School of Medicine, Yale University, New Haven, CT, USA

V1 First published: 13 Nov 2017, 6:1998

https://doi.org/10.12688/f1000research.12776.1

Second version: 20 Feb 2018, 6:1998

https://doi.org/10.12688/f1000research.12776.2

Latest published: 28 Mar 2018, 6:1998

https://doi.org/10.12688/f1000research.12776.3

\section{Abstract}

Infective endocarditis (IE) is a serious and life threatening cardiac condition, most commonly caused by staphylococci, streptococci, enterococci and rarely by HACEK organisms (Haemophilus,

Aggregatibacter, Cardiobacterium, Eikenella corrodens and Kingella). Here, we present a case of IE caused by Aerococcus urinae in a 75-yearold man with a bioprosthetic aortic valve. Aerococcus urinae is a grampositive, catalase negative microorganism, and is usually an isolate of complicated urinary tract infections in the elderly male population. It is associated with high morbidity and mortality. Awareness of this organism as a cause of IE is important, since failure to recognize the condition may lead to adverse clinical outcomes and significant complications with even fatal outcome, as in this case.

Keywords

infective endocarditis, prosthetic valve endocarditis, Aerococcus urinae

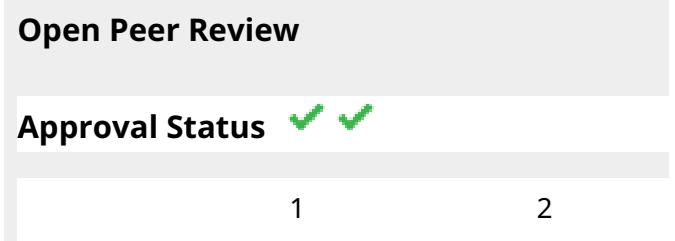

version 3
(revision)
28 Mar 2018
version 2
(revision)

1. Magnus Rasmussen, Lund University, Lund, Sweden

2. Jens J Christensen, Slagelse Hospital, Slagelse, Denmark Any reports and responses or comments on the article can be found at the end of the article. 
Corresponding author: Carina Dehner (verdaderamente88@gmail.com)

Author roles: Adeel M: Conceptualization, Data Curation, Investigation, Methodology, Validation, Writing - Original Draft Preparation; Tariq S: Investigation, Methodology; Akthar H: Conceptualization, Writing - Review \& Editing; Zaghloul A: Methodology, Writing -

Review \& Editing; Iorgoveanu C: Methodology, Supervision, Writing - Review \& Editing; Dehner C: Conceptualization, Formal Analysis, Investigation, Software, Writing - Review \& Editing

Competing interests: No competing interests were disclosed.

Grant information: The author(s) declared that no grants were involved in supporting this work.

Copyright: ( 2017 Adeel $\mathrm{M}$ et al. This is an open access article distributed under the terms of the Creative Commons Attribution License, which permits unrestricted use, distribution, and reproduction in any medium, provided the original work is properly cited.

How to cite this article: Adeel M, Tariq S, Akthar $\mathrm{H}$ et al. Case Report: A rare case of prosthetic valve infective endocarditis caused by Aerococcus urinae [version 1; peer review: 2 approved with reservations] F1000Research 2017, 6:1998

https://doi.org/10.12688/f1000research.12776.1

First published: 13 Nov 2017, 6:1998 https://doi.org/10.12688/f1000research.12776.1 


\section{Introduction}

The diagnosis of infective endocarditis (Habib et al.) is based on a number of factors, including patient history, physical examination, as well as diagnostic tools (blood cultures, chest X-ray and echocardiography) (Durack et al., 1994), (Lukes et al., 1993). Risk factors for IE include advanced age ( $>60$ years), male gender, history of intravenous drug use, poor dentition, structural or valvular heart disease and presence of prosthesis. It is most commonly caused by Staphylococcus aureus, Streptococcus viridans, and enterococci, and rarely by HACEK (Sharara et al., 2016) organisms. Here, we describe a rare case of IE secondary to Aerococcus urinae, a gram-positive, catalase negative coccus that grows in clusters. It is associated with high mortality and neurological complications (Ebnother et al., 2002).

\section{Case report}

A 75-year-old Caucasian man presented to his local hospital with malaise, fever and nausea for 5 days. He had a bio prosthetic aortic valve replacement for mixed aortic valve disease 12 years ago, further significant past medical history included placement of a permanent pacemaker for complete heart block, right total hip replacement, hypertension and benign prostatic hyperplasia (BPH). The patient had no history of smoking, alcohol consumption or illicit drug use. The patient had no recent surgeries or dental work and the review of systems was unremarkable. The physical exam revealed vital parameters of HR 97 bpm regular, BP $134 / 87$, temperature of $101.5^{\circ} \mathrm{F}$, respiratory rate of 18 per minute and oxygen saturation of $96 \%$ on room air. On precordial auscultation a systolic and a diastolic murmur were heard in aortic area, mild bi-basal crepitation, but no JVD or peripheral edema. The rest of the physical exam was unremarkable. His labs showed a normal white cell count (WCC) of $9.9 \times 10^{6} \mathrm{~L}$, but his C-reactive protein (CRP) was elevated to 214.9 (normal $<5 \mathrm{mg} / \mathrm{L}$ ) with a stable haemoglobin $(11.2 \mathrm{~g} / \mathrm{dl})$, further labs were unremarkable. His mid-stream urine showed $\mathrm{WCC}<20$; red cell count (RCC) of 20-50 and it grew mixed organisms, all considered part of the normal flora. Chest X-ray, CT scan of the brain, thorax, abdomen and pelvis did not show any significant cause of sepsis.

The patient was empirically commenced on IV piperacillintazobactam and vancomycin for sepsis treatment. His blood cultures grew Aerococcus urinae sensitive to penicillin within 24 hours of admission.

A trans-thoracic echocardiogram showed mild aortic regurgitation and mitral regurgitation with no clear vegetation, however, trans-esophageal echocardiogram (TOE) showed a moderate aortic regurgitation due to a large mobile vegetation on the bio-prosthetic aortic valve with normal left ventricular function, no peri-valvular abscess was noted (See Image 1a and 1b).

Clinical presentation, echocardiographic findings and positive blood cultures fulfilled Duke's criteria (Hoen et al., 1996) for IE. Patient was managed as prosthetic aortic valve endocarditis from Aerococcus urinae with IV amoxicillin 2 grams every 4 hours, and gentamicin $1 \mathrm{mg} / \mathrm{kg}$ twice daily as per local guidelines. Antibiotic
A

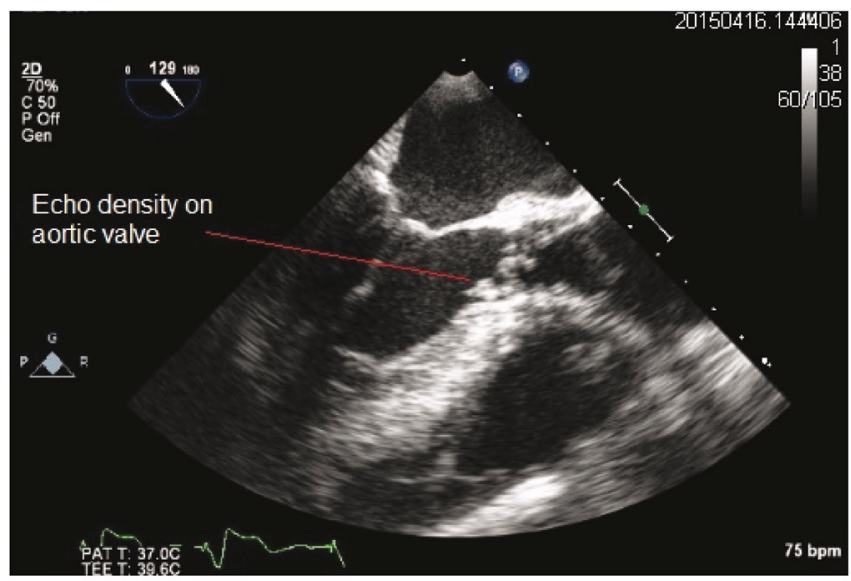

B

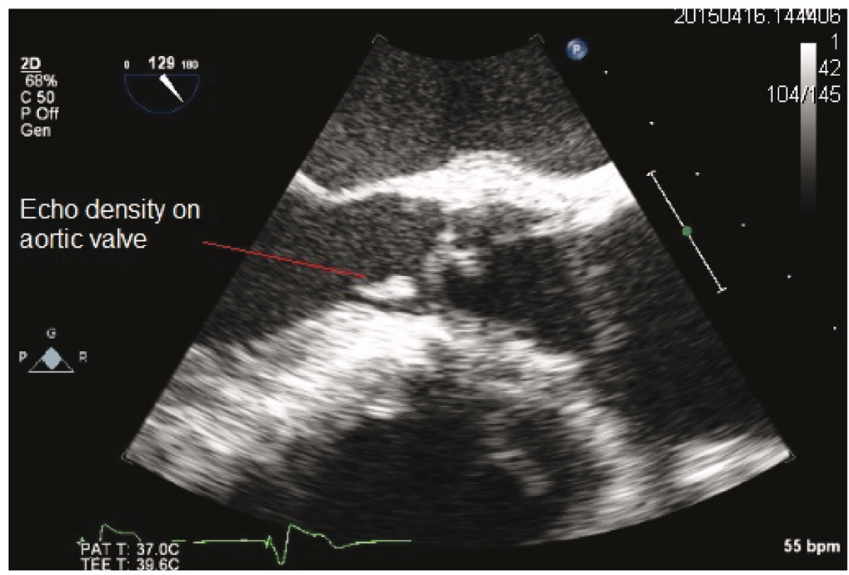

Figure 1. 1A: Transesophageal echocardiogram (TEE), midesophageal view showing mobile echo density on prosthetic aortic valve. 1B: Transesophageal echocardiogram (TEE), mid-esophageal view enlarged to show mobile echo density on prosthetic aortic valve.

therapy for 6 weeks in total with early surgery for prosthetic valve replacement was planned (Truninger et al., 1999).

Despite prompt initiation of appropriate antibiotic treatment and intensive clinical monitoring, the patient failed to improve this hospitalization and developed a large pulmonary edema and progressive aortic regurgitation, and died before definitive surgery. As per family's wishes, an autopsy was not performed.

\section{Discussion}

Aerococcus urinae is a gram-positive, catalase negative coccus which grows in clusters. It is mostly associated with urinary tract infections in elderly men, especially in the setting of structural abnormalities e.g. BPH, urethral strictures and nephrolithiasis. It has been associated with culture negative infective endocarditis (Slany et al., 2007). It is reported to be sensitive to penicillins/ 
cephalosporins and resistant to sulfonamides and aminoglycosides (Skov et al., 2001). There are less than 20 reported cases of IE caused by Aerococcus urinae worldwide.

Despite being sensitive to common antibiotics, prosthetic valve endocarditis (PVE) secondary to Aerococcus urinae can be difficult to manage with antibiotic therapy alone, and often requires surgical intervention (Wang et al., 2007). The indications for surgical intervention for PVE include severe prosthetic dysfunction, severe heart failure, large persistent vegetation and abscess or peri-valvular involvement (Habib et al., 2005). The presence of vegetation on the valve created a consistent source of bacteria that could embolize and can serve as a source of sepsis.

This case highlights the importance of source control by expediting prosthesis removal in presence of overt symptoms of worsening cardiac failure and worsening prosthesis dysfunction (regurgitation in this case), as medical therapy alone may not be sufficient to effectively treat Aerococcus urinae IE despite appropriate sensitivities. Early identification is crucial and can be life-saving. The main problem is current diagnostic testing for microorganisms - whereas 16 s sequencing would be the most time-efficient method, it's rarely done, as the expertise is limited and costs are high. Recently, there is good evidence for the use of MALDI-TOF (Senneby et al., 2013), (Senneby et al., 2016) due to increased detection rates, even in direct comparison to $16 \mathrm{~s}$ sequencing.

In conclusion, Aerococcus urinae used to be a rare cause of IE but rates have been increasing significantly within the last 10 years. Therefore establishing a concise and broadly acknowledged protocol from diagnosis up to patient management is critical.

\section{Consent}

Written informed consent for publication of their clinical details was obtained from the patient. Permission was also granted from a next of kin for publication of the manuscript.

\section{Competing interests}

No competing interests were disclosed.

\section{Grant information}

The author(s) declared that no grants were involved in supporting this work.
Durack DT, Lukes AS, Bright DK: New criteria for diagnosis of infective endocarditis: utilization of specific echocardiographic findings. Duke Endocarditis Service. Am J Med. 1994; 96(3): 200-209. PubMed Abstract | Publisher Full Text

Ebnöther C, Altwegg M, Gottschalk J, et al.: Aerococcus urinae endocarditis: case report and review of the literature. Infection. 2002; 30(5): 310-313. PubMed Abstract | Publisher Full Text

Habib G, Tribouilloy C, Thuny F, et al.: Prosthetic valve endocarditis: who needs surgery? A multicentre study of 104 cases. Heart. 2005; 91(7): 954-959. PubMed Abstract | Publisher Full Text | Free Full Text

Hoen B, Béguinot I, Rabaud C, et al:: The Duke criteria for diagnosing infective endocarditis are specific: analysis of 100 patients with acute fever or fever of unknown origin. Clin Infect Dis. 1996; 23(2): 298-302.

PubMed Abstract | Publisher Full Text

Lukes AS, Bright DK, Durack DT: Diagnosis of infective endocarditis. Infect Dis Clin North Am. 1993; 7(1): 1-8.

PubMed Abstract

Senneby E, Göransson L, Weiber S, et al:: A population-based study of aerococcal bacteraemia in the MALDI-TOF MS-era. Eur J Clin Microbiol Infect Dis. 2016; 35(5): 755-762.

PubMed Abstract | Publisher Full Text
Senneby E, Nilson B, Petersson AC, et al:: Matrix-assisted laser desorption ionization-time of flight mass spectrometry is a sensitive and specific method for identification of aerococci. J Clin Microbiol. 2013; 51(4): 1303-1304. PubMed Abstract | Publisher Full Text | Free Full Text

Sharara SL, Tayyar R, Kanafani ZA, et al.: HACEK endocarditis: a review. Expert Rev Anti Infect Ther. 2016; 14(6): 539-545.

PubMed Abstract| Publisher FullText

Skov R, Christensen JJ, Korner B, et al.: In vitro antimicrobial susceptibility of Aerococcus urinae to 14 antibiotics, and time-kill curves for penicillin, gentamicin and vancomycin. J Antimicrob Chemother. 2001; 48(5): 653-658. PubMed Abstract | Publisher Full Text

Slany M, Freiberger T, Pavlik $\mathrm{P}$, et al:: Culture-negative infective endocarditis caused by Aerococcus urinae. J Heart Valve Dis. 2007; 16(2): 203-205. PubMed Abstract

Truninger K, Attenhofer Jost $\mathrm{CH}$, Seifert B, et al.: Long term follow up of prosthetic valve endocarditis: what characteristics identify patients who were treated successfully with antibiotics alone? Heart. 1999; 82(6): 714-720. PubMed Abstract | Publisher Full Text | Free Full Text

Wang A, Athan E, Pappas PA, et al: Contemporary clinical profile and outcome of prosthetic valve endocarditis. JAMA. 2007; 297(12): 1354-1361.

PubMed Abstract | Publisher Full Text 


\section{Open Peer Review}

\section{Current Peer Review Status: ? ?}

\section{Version 1}

Reviewer Report 22 January 2018

https://doi.org/10.5256/f1000research.13841.r29616

(C) 2018 Christensen J. This is an open access peer review report distributed under the terms of the Creative Commons Attribution License, which permits unrestricted use, distribution, and reproduction in any medium, provided the original work is properly cited.

\section{Jens J Christensen}

Department of Clinical Microbiology, Slagelse Hospital, Slagelse, Denmark

A fatal case of IE caused by Aerococcus urinae, in a 75-yearold man with a bioprosthetic aortic valve is presented and discussed. Very precise and covering comments have been given by reviewer 1 . Microbiological data should be examined thouroughly and extended. Language correction seems indicated. The following comments can be added.

1) Abstract:

It is always important to also having focus on more rare etiologies of IE. In the abstract it is stated that the mortality rate is high. This is suggested to be modified to: Initial descriptions of collections of IE cases with A. urinae demonstrated a high morbidity and mortality rate, whereas a recent Swedish epidemiological study could not retrieve this.

2) Introduction:

Dukes criteria should be mentioned.

There is not a species named Streptococcus viridans.

Recent diagnostic improvements should be included, especially MALDI-TOF mass spectrometry.

a gram-positive, catalase-negative

3) Case description:

Specific description of PM electrode findings should be given.

A more detailed disease timespan is desirable.

Microbiological data are very scarce. Blood-culture system and number of positive bottles should be given. Likewise identification criteria and susceptibility methods and results, including MIC values of relevant antibiotics should be given.

A thorough microbiological examination of the manuscript seems indicated

Aerococcus urinae should only be fully written the first time

4) Discussion 
$16 \mathrm{~S}$ is slang: it should be partial $16 \mathrm{~S}$ rRNA gene sequencing analysis

Is the background of the case's history and progression described in sufficient detail? Partly

Are enough details provided of any physical examination and diagnostic tests, treatment given and outcomes?

Partly

Is sufficient discussion included of the importance of the findings and their relevance to future understanding of disease processes, diagnosis or treatment?

Partly

Is the case presented with sufficient detail to be useful for other practitioners?

No

Competing Interests: No competing interests were disclosed.

Reviewer Expertise: Clinical microbiology

I confirm that I have read this submission and believe that I have an appropriate level of expertise to confirm that it is of an acceptable scientific standard, however I have significant reservations, as outlined above.

Author Response 13 Feb 2018

Carina Dehner, Yale University, New Haven, USA

1. The prognosis of $A$. urinae IE is not poor. Many cases with fatal outcome have been published but in the only population-based survey ${ }^{1}$ demonstrate a relatively favourable prognosis compared to other pathogens. The risk with case reports is a publication-bias where only dramatic cases are published. The case series should be quoted and is the only reliable source on information on $A$. urinae IE. A poor prognosis is claimed in the abstract, introduction and discussion. This claim must be modified based on the findings by Sunnerhagen et al.

A new reference was added to the introduction about favourable outcome to IE caused by Aerococcus urinae

2. A diagnosis of IE is established through the Dukes criteria, I suggest a reference to $\mathrm{Li}$ is given ${ }^{2}$.

Symptoms and chest X-ray are irrelevant for the diagnostic process. Please modify introduction.

A new reference was added to diagnose IE based on Dukes criteria or their modifications 
3. In the case description it is twice stated that the patient has sepsis. Sepsis-3 criteria are not fulfilled. Please rephrase.

\section{Rephrased}

4. The cultures grew A. urinae. How was the species determination performed? How many cultures? MIC for ampicillin and gentamycin should be given.

\section{MICs for ampicillin and gentamicin added, description added about blood cultures}

5. It is claimed that TEE demonstrates a "moderate aortic regurgitation due to a large mobile vegetation". It is important if the regurgitation was paravalvular or through the valves. Maximum size of the vegetation is also crucial since this is important for establishing the indication for operation. Left ventricular function should also be commented on as well as if there were signs of vegetations on the pacemaker cable.

Transesophgeal echo description expanded, commented on LV and pacemaker lead.

6. It is claimed that ampi+genta was commenced according to local guidelines. For which bacterial species are these guidelines meant. The use of aminoglycosides in this condition is controversial ${ }^{1}$.

\section{Hospital guidelines for suspected/possible IE were followed}

7. How was "progressive aortic regurgitation verified? Could pacemaker failure have played a role?

\section{Repeat transthoracic echo showed worsening of regurgitation; this is added to the case}

8. Why was the patient not moved for emergency surgery when he deteriorated? This seem like an avoidable fatality!

\section{Deterioration was sudden and rapid, arrangements were made but patient died before the surgery}

9. In the discussion it is claimed that there are only 20 reports. This is not true ${ }^{1}$. Cases up until 2015 are summarized in a review ${ }^{3}$.

\section{Updated number of Aerococcus urinae IE reported}

10. Surgical intervention is claimed to be common in the discussion with a quote to Wang. Please read and quote Sunnerhagen instead ${ }^{1}$. Surgery is relatively rarely needed.

Reference added to show that surgery in most cases is not indicated 
11. "Large persistent vegetation" is claimed as an indication for surgery. Large is enough.

\section{Modified}

12 "The presence of vegetation on the valve created a consistent source of bacteria that could embolize and can serve as a source of sepsis." This statement has nothing to do with the current case and should be omitted.

\section{Omitted}

13 "The main problem is current diagnostic testing for microorganisms- whereas $16 \mathrm{~s}$ sequencing would be the most time-efficient method, it's rarely done, as the expertise is limited and costs... and so on" This is irrelevant for the case since the reason to operate is not dependent on microbiological diagnostics. Irrespective of the causative pathogen this patient would have been saved by timely heart surgery.

\section{This is kept; we wish our readers to know that improved methods of isolation are important and could help with management}

14. The claim "In conclusion, Aerococcus urinae used to be a rare cause of IE but rates have been increasing significantly within the last 10 years." Lacks support and should be deleted. $A$. urinae has been increasingly REPORTED as a cause of IE but incidence is likely unchanged.

This is now added that that increase in reported cases is due to better isolation methods.

15. In discussing Duke criteria in the case presentation one must keep in mind that $A$. urinae in $2 / 2$ cultures ( $4 / 4$ bottles) only fulfill Duke criteria if the cultures were taken with

\section{Agreed}

Competing Interests: No competing interests were disclosed.

Reviewer Report 11 December 2017

https://doi.org/10.5256/f1000research.13841.r28819

(c) 2017 Rasmussen M. This is an open access peer review report distributed under the terms of the Creative Commons Attribution License, which permits unrestricted use, distribution, and reproduction in any medium, provided the original work is properly cited.

Magnus Rasmussen 
Department of Clinical Sciences, Division of Infection Medicine, Faculty of Medicine, Lund University, Lund, Sweden

This work describes a case of prosthetic valve infective (IE) caused by Aerococcus urinae with fatal outcome. The number of case reports on this condition is increasing and it is not immediately obvious that another case with poor outcome is helpful. This case, however, has an important learning point in that a patient with prosthetic valve endocarditis, in resource-rich settings, must be treated in a centre where acute cardiac surgery can be performed or near such a centre. It is of less importance if the causative bacterium in this case were A. urinae or any other bacterium. I list my major concerns and minor points below:

Major concerns

1. The prognosis of $A$. urinae IE is not poor. Many cases with fatal outcome have been published but in the only population-based survey ${ }^{1}$ demonstrate a relatively favourable prognosis compared to other pathogens. The risk with case reports is a publication-bias where only dramatic cases are published. The case series should be quoted and is the only reliable source on information on $A$. urinae IE. A poor prognosis is claimed in the abstract, introduction and discussion. This claim must be modified based on the findings by Sunnerhagen et al.

2. A diagnosis of IE is established through the Dukes criteria, I suggest a reference to $\mathrm{Li}$ is given ${ }^{2}$. Symptoms and chest $X$-ray are irrelevant for the diagnostic process. Please modify introduction.

3. In the case description it is twice stated that the patient has sepsis. Sepsis-3 criteria are not fulfilled. Please rephrase.

4. The cultures grew $A$. urinae. How was the species determination performed? How many cultures? MIC for ampicillin and gentamycin should be given.

5. It is claimed that TEE demonstrates a "moderate aortic regurgitation due to a large mobile vegetation". It is important if the regurgitation was paravalvular or through the valves. Maximum size of the vegetation is also crucial since this is important for establishing the indication for operation. Left ventricular function should also be commented on as well as if there were signs of vegetations on the pacemaker cable.

6. It is claimed that ampi+genta was commenced according to local guidelines. For which bacterial species are these guidelines meant. The use of aminoglycosides in this condition is controversial ${ }^{1}$.

7. How was "progressive aortic regurgitation verified? Could pacemaker failure have played a role? 8 . Why was the patient not moved for emergency surgery when he deteriorated? This seem like an avoidable fatality!

9. In the discussion it is claimed that there are only 20 reports. This is not true ${ }^{1}$. Cases up until 2015 are summarized in a review ${ }^{3}$.

10. Surgical intervention is claimed to be common in the discussion with a quote to Wang. Please read and quote Sunnerhagen instead ${ }^{1}$. Surgery is relatively rarely needed.

11. "Large persistent vegetation" is claimed as an indication for surgery. Large is enough.

12 "The presence of vegetation on the valve created a consistent source of bacteria that could embolize and can serve as a source of sepsis." This statement has nothing to do with the current case and should be omitted.

13 “The main problem is current diagnostic testing for microorganisms- whereas 16 s sequencing would be the most time-efficient method, it's rarely done, as the expertise is limited and costs... and so on" This is irrelevant for the case since the reason to operate is not dependent on microbiological diagnostics. Irrespective of the causative pathogen this patient would have been saved by timely heart surgery.

14. The claim "In conclusion, Aerococcus urinae used to be a rare cause of IE but 
rates have been increasing significantly within the last 10 years." Lacks support and should be deleted. A. urinae has been increasingly REPORTED as a cause of IE but incidence is likely unchanged.

15. In discussing Duke criteria in the case presentation one must keep in mind that $A$. urinae in $2 / 2$ cultures (4/4 bottles) only fulfill Duke criteria if the cultures were taken with

Minor comments

1. I suggest another title. Something like "fatal case of A. urinae prosthetic valve endocrditis."

2. Why mention HACEK in the abstract? Those organisms are exceedingly rare and for example much less common than betaheamolytic strep.

3. In case presentation spell out JVD.

4. "Stable haemoglobin"- what is meant. Are the authors referring to repeated measurements?

\section{References}

1. Sunnerhagen T, Nilson B, Olaison L, Rasmussen M: Clinical and microbiological features of infective endocarditis caused by aerococci.Infection. 2016; 44 (2): 167-73 PubMed Abstract | Publisher Full Text

2. Li JS, Sexton DJ, Mick N, Nettles R, et al.: Proposed modifications to the Duke criteria for the diagnosis of infective endocarditis.Clin Infect Dis. 2000; 30 (4): 633-8 PubMed Abstract | Publisher Full Text

3. Rasmussen M: Aerococcus: an increasingly acknowledged human pathogen.Clin Microbiol Infect. 2016; 22 (1): 22-27 PubMed Abstract | Publisher Full Text

Is the background of the case's history and progression described in sufficient detail? Partly

Are enough details provided of any physical examination and diagnostic tests, treatment given and outcomes?

Partly

Is sufficient discussion included of the importance of the findings and their relevance to future understanding of disease processes, diagnosis or treatment?

No

Is the case presented with sufficient detail to be useful for other practitioners? Partly

Competing Interests: No competing interests were disclosed.

Reviewer Expertise: endocarditis

I confirm that I have read this submission and believe that I have an appropriate level of expertise to confirm that it is of an acceptable scientific standard, however I have significant reservations, as outlined above. 


\section{Author Response 13 Feb 2018}

Carina Dehner, Yale University, New Haven, USA

1) Abstract:

- It is always important to also having focus on more rare etiologies of IE. In the abstract it is stated that the mortality rate is high. This is suggested to be modified to: Initial descriptions of collections of IE cases with A. urinae demonstrated a high morbidity and mortality rate, whereas a recent Swedish epidemiological study could not retrieve this.

New reference to the introduction was added to highlight the better outcome

2) Introduction:

- Dukes criteria should be mentioned.

- New references added to mention Duke's criteria or its modifications

- There is not a species named Streptococcus viridans.

\section{Correction made}

- $\quad$ Recent diagnostic improvements should be included, especially MALDI-TOF mass spectrometry.

Added in abstract

- a gram-positive, catalase-negative

\section{Correction made}

3) Case description: Specific description of PM electrode findings should be given.

\section{Included in description, PM lead was not involved.}

Microbiological data are very scarce. Blood-culture system and number of positive bottles should be given. Likewise identification criteria and susceptibility methods and results, including MIC values of relevant antibiotics should be given.

\section{This is now added to the case description}

4) Discussion: $16 \mathrm{~S}$ is slang: it should be partial 16S rRNA gene sequencing analysis Correction made

Competing Interests: No competing interests were disclosed. 
The benefits of publishing with F1000Research:

- Your article is published within days, with no editorial bias

- You can publish traditional articles, null/negative results, case reports, data notes and more

- The peer review process is transparent and collaborative

- Your article is indexed in PubMed after passing peer review

- Dedicated customer support at every stage

For pre-submission enquiries, contact research@f1000.com 